\title{
Gold Nanoparticles Uptake in Monoculture vs Co-culture of Pancreatic Cancer Cells
}

\author{
Abdulaziz Alhussan ${ }^{1}$, Julian Smazynski ${ }^{2}$, Devika Chithrani ${ }^{1,2}$ \\ ${ }^{1}$ Department of Physics and Astronomy, University of Victoria, Victoria, BC V8P 5C2, Canada. \\ alhussan@uvic.ca; devikac@uvic.ca \\ ${ }^{2}$ British Columbia Cancer-Victoria, Victoria, BC V8R 6V5, Canada. \\ jsmazynski@bccrc.ca
}

\begin{abstract}
Despite the advancements in medicine in the last decade, pancreatic cancer is still one of the deadliest types of cancers with a survival rate of less than $7 \%$. The location of pancreas, late detection, the metastatic nature of the disease, and the condensed desmoplasia and stroma of the tumour mass render existing therapeutics inadequate for many patients. Nanotechnology has emerged as a possible solution for hard-to-treat cancers. High atomic number materials such as gold nanoparticles (GNPs) have radiosensitizing properties and could also be used as a drug carrier to precisely deliver drugs to their target. Most recent studies have focused on the radiosensitizing effects of gold in monocultures of cancer cells. However, co-culture systems differ from monoculture systems in the cell-to-cell intercommunication between the different cell lines used. Cancer associated fibroblasts (CAFs) are another very important components of the tumour microenvironment that plays a negative role in radio- and chemo- resistance. They communicate directly with cancer cells to help promote tumour growth. In this paper, separation of cancer cells from CAFs, and GNPs uptake in co-cultures of cancer cells and CAFs vs monocultures were investigated. Successful separation between the two cell lines used was attainable with high accuracy using magnetic beads separation. Our data show that GNP uptakes is roughly the same between cells of the same type whether grown in monoculture or in co-culture. The results also reveal a much higher uptake of GNPs by CAFs compared to tumour cells. We conclude that targeting tumour cells and CAFs in co-culture using radiosensitizing GNPs is feasible and is expected to yield encouraging outcomes.
\end{abstract}

Keywords: MiaPaca-2, Cancer Associated Fibroblasts, Gold Nanoparticles, Pancreatic Cancer, Co-culture.

\section{Introduction}

There are over a hundred type of cancer of which pancreatic ductal adenocarcinoma (PDAC) is one of the most lethal cancers standing in 4th place in terms of number of deaths with a 5-year death rate of higher than $90 \%$ and the majority of patients dying within the first year [1-2]. Current treatments modalities such as surgery and radiotherapy are limited by metastases and normal tissue toxicity, respectively [3-5]. The presence of the dense tumour microenvironment (TME) in PDAC, which includes cancer associated fibroblasts (CAFs), and the extracellular matrix (ECM), prevents propagation of chemotherapeutic drugs denying the delivery of the necessary doses [6-7]. The relationship between cancer cells and CAFs is mutual in which cancer cells recruit normal cells, turning them into CAFs to support the development of the tumour, while CAFs secret several growth factors, enzymes and cytokines that promote the proliferation of the tumour, help with metastasis of tumour cells, and weaken the impact of the immune system on tumour cells [8-11]. Consequently, the intercommunication between cancer cells and CAFs fosters the ideal niche for tumour to develop. Therefore, developing new technologies that target both tumour cells and CAFs is essential. Nanoparticles of high-Z materials such as gold nanoparticles (GNPs) have shown promising results as radiosensitizer agents in radiotherapy and as vectors for targeted-drug delivery in chemotherapy $[4-5,12]$. There is also tremendous evidence that GNPs and low concentrations of anticancer drugs can sensitize cancer cells to radiotherapy in monoculture in vitro environment [13-17]. In monoculture, CAFs have shown to uptake more GNPs into them when compared to cancer cells [16-17]. However, real-life tumour environments exist in co-culture and not in monocultures. The main problem with monoculture structures is the lack of intercommunications between cancer cells and CAFs, thus, it is necessary to better represent cell-cell interactions in these TME by creating co-culture systems which may affect the uptake of GNPs into each cell line [18-21]. Despite the importance of co-culture experiments and its advantages over monoculture experiments, the uptake of GNPs in co-culture of cells of pancreatic origin has not been investigated. Understanding the interactions of cells in co-cultures with treatment modalities is critical in reaping the benefits of nanotechnology especially for pancreatic cancer. Insight on the interactions of GNPs with cancer cells and CAFs in co- 
culture systems will lay a roadmap to doing more in-depth co-culture experiments before transitioning to 3D in vitro coculture systems and then to in vivo models. One of the problems that arises when using GNP in a co-culture environment is the dilemma of accurately quantifying the uptake of GNP in each cell line used due to the difficulty of separating the cell lines used for precise analysis. Therefore, this paper sheds the light on the behaviour of GNPs in a co-culture environment of cancer cells and CAFs by addressing two important questions:

1. Is it possible to separate between cancer cells and CAFs of pancreatic origin when the cells are grown in a coculture environment and recover them for analysis?

2. How different is GNPs uptake between cancer cells grown in a monoculture environment vs cancer cells grown in a co-culture environment? and between CAFs grown in a monoculture environment and CAFs grown in a co-culture environment?

\section{Results \& Discussion}

Citrate reduction method was used to produce gold nanoparticles (GNPs) of $13.2 \pm 1.3 \mathrm{~nm}$ diameter in size. Gold nanoparticles of sizes 10-15 $\mathrm{nm}$ has shown better penetration and uptake capabilities in 3D tumour models [18]. Polyethylene glycol (PEG) and RGD peptides were used at a surface density of 1 PEG per $\mathrm{nm}^{2}$ of the GNP surface area and at one molecule of RGD for every two PEG molecules to functionalize the negatively charged GNPs to create GNPPEG-RGD, Fig.1A. The former was added to simulate an in vivo environment in which PEG would help nanoparticles avoid the immune system, and the latter was added to improve the nanoparticles' uptake into cancer cells [16-17]. GNPs shape and size were verified using Transmission Electron Microscopy (TEM), Fig.1B. Co-culture cells were cultured together for a period of $72 \mathrm{hrs}$ to ensure enough time for intercommunication between cancer cells and CAFs [22]. All cells were dosed with $7.5 \mu \mathrm{g} / \mathrm{mL}$ of GNPPEG-RGD post-incubation for $24 \mathrm{hrs}$.

A

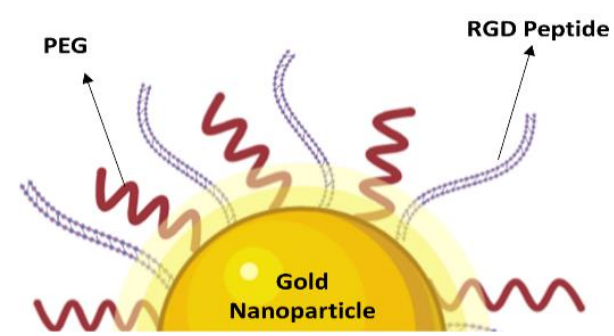

B

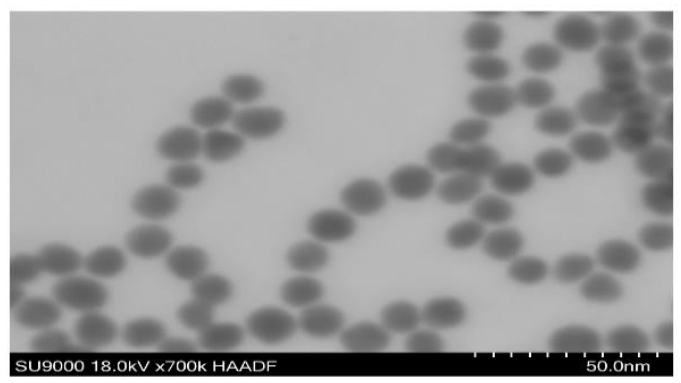

Fig. 1: Characterization of the gold nanoparticles (GNPs). A) Schematic showing GNP with measured core diameter of $\sim 13.2$ functionalized with a polyethylene glycol (PEG) molecules as stabilizing agents, and RGD peptides to improve GNPs uptake. B) Transmission Electron Microscopy (TEM) image of GNP.

To be able to quantify the number of nanoparticles per cell precisely using methods such as Inductively Coupled Plasma-Mass Spectrometry (ICP-MS) it is important to separate specific cell populations from a heterogeneous microenvironment of cells for analysis. A successful cell isolation or cell sorting would allow us to measure the effects of cell-to-cell interactions on the uptake of nanoparticles in the different cell lines used. We have explored two ways of separating between cancer cells from CAFs, separating the cells by filtration, and separating cells using magnetic beads. We used two pancreatic cell lines, Panc1 and MiaPaca2, and one fibroblasts of pancreatic origin CAF98. Cell separation by filtration, which is based on cell size, was inconclusive, this might be due to the variation of cell sizes in the cell populations of the cell lines used. On the other hand, the magnetic beads cell sorting methods, which involves targeting cells using antibodies or ligands directed against specific cell surface antigens, yielded very good results for the separation for the MiaPaca2/CAF98 co-culture but not for the Panc1/CAF98 co-culture. It seemed like the anti-fibroblast antibody bound strongly to specific antigens on Panc1 cancer cell line. This could be because Panc1 expresses the antigen that the anti-fibroblast antibody recognizes. This problem was not evident for the MiaPaca2/CAF98 co-culture as illustrated in Fig.2A, where a successful cell sorting and recovery of cells was achieved. Over 95\% of cells in the isolated tumour portion of cells after separation from the MiaPaca2/CAF98 co-culture being MiaPaca2 and the same high percentage was achieved for the fraction of CAF98 cells in the isolated CAFs portion. We have also looked at the uptake of FITC labelled GNPs in monocultures of MiaPaca2 and CAF98, vs the uptake of GNPs in MiaPaca2 and 
CAF98 when they are in co-culture through flow cytometry Fig.2B. No significant difference was found between the average uptake of FITC-labelled GNPs in MiaPaca2 and CAF98 when they are in monoculture vs when they are in co-culture. However, the uptake of GNPs in CAFs is much higher than that of tumour cells in both monoculture and co-culture, which is in agreement with our previous experiments that were done in monoculture [16-17]. This is an important observation that might open the door for targeting both tumour cells and CAFs using GNPs.

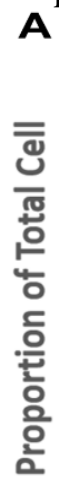

A

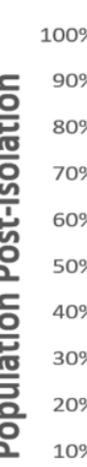

$10 \%$ $0 \%$

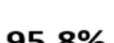

95.8\%

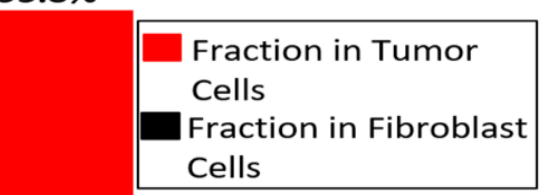

MiaPaca2
B

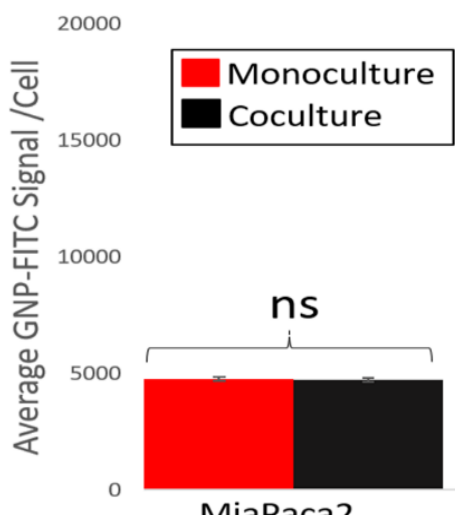

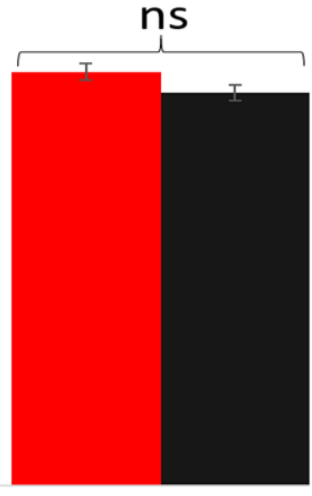

CAFs

Fig. 2: MiaPaca2 vs CAFs Flow Cytometry Data. (A) Fractions of MiaPaca2 and CAFs populations post-isolation from co-culture in tumour cells portion vs in fibroblasts portion. (B) Average GNP-FITC signal per cell of MiaPaca2 and CAFs in monoculture vs coculture; ns indicates non-significance.

Additionally, GNPs distribution in the cells was mapped qualitatively using confocal microscopy Fig.3A and darkfield coupled with hyperspectral imaging (HIS), Fig.3B. Similar to the flow cytometry data, cells were incubated with $G N P_{P E G-}$ $R G D$ complex at a concentration of $7.5 \mu \mathrm{g} / \mathrm{mL}$ for $24 \mathrm{hrs}$. This concentration of GNPs is within adequate doses for in vivo and clinical applications [23]. Both confocal and darkfield solidify our quantitative data and barely show any different in the uptake of GNPs in tumour cells and CAFs in co-cultures vs in monocultures. They also clearly show a higher uptake of GNPs in CAFs compared to in cancer cells. The existence of GNPs in cells was verified using spectral mapping feature of HSI, as illustrated in the rightmost top corner of Fig.3B where GNP spectra were collected from bright spectra from within cells confirming that they are from gold from when compared to available data in the imaging library.
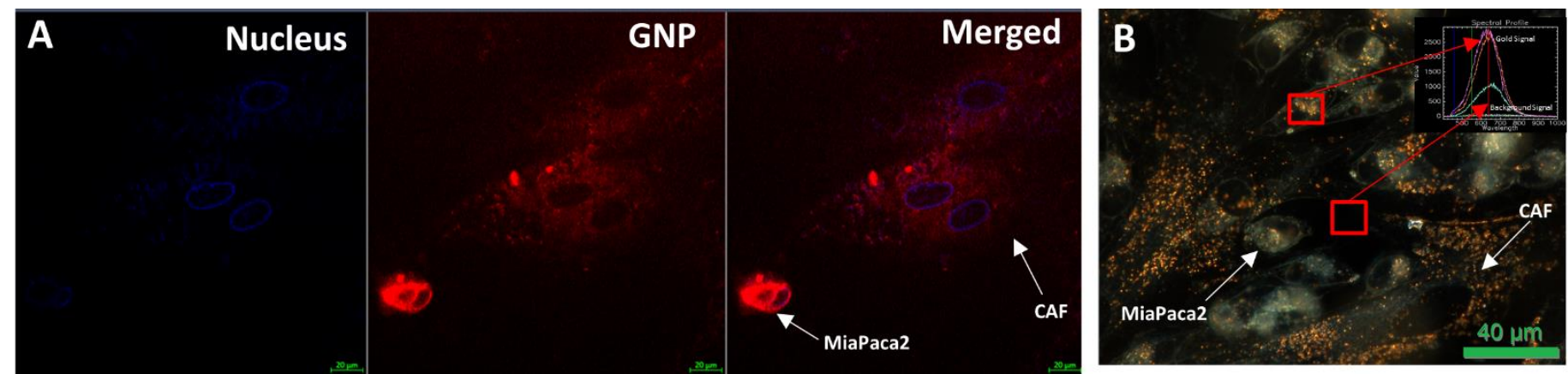

Fig. 3: Gold Nanoparticles (GNPs) uptake in cancer cells and CAFs in monoculture vs co-culture. (A) Visualization of GNP distribution in co-culture of MiaPaca2 and CAFs using confocal imaging where first column shows nucleus, second column shows GNPs, third column shows both merged. (B) shows darkfield microscopy of co-culture of MiaPaca2/CAFs. Overlay: Hyperspectral imaging (HSI) which shows a spectrum collected from GNP clusters. Scale bars are $20 \mu \mathrm{m}$ and $40 \mu \mathrm{m}$ for confocal and DF, respectively

\section{Conclusion}

The co-culture cell separation of cancer cells from CAFs is achievable with acceptable precision using magnetic beads isolation, where cells can then be recovered for further analysis. The uptake of GNPs in CAFs and in cancer cells in monoculture was roughly the same as their uptake in CAFs and in cancer cells, respectively, when grown in co-culture. The results also show a considerably higher uptake of GNP in CAFs compared to cancer cells in co-coculture consistent with our results from previous monoculture work. The results indicate the possibility of targeting both cancer cells and CAFs very 
successfully using GNPs radiosensitizers in a co-culture environment. This form of new therapeutic is necessary in treating hard-to-treat cancers such as pancreatic cancer.

\section{Acknowledgements}

The authors would like to acknowledge the support from University of British Columbia, BC Cancer (Vancouver \&Victoria). This study was funded by a Nanomedicines Innovation Network Strategic Initiative fund (NMIN-SI) from government of Canada, the John R. Evans Leaders Fund (JELF) from the Canada Foundation for Innovation (CFI) and British Columbia Knowledge Development Fund (BCKDF), the NSERC Discovery grant from the Natural Sciences and Engineering Research Council of Canada (NSERC), and a collaborative health grant from the University of Victoria.

\section{References}

[1] Orth, M., Metzger, P., Gerum, S., Mayerle, J., Schneider, G., Belka, C., Schnurr, M., \& Lauber, K. (2019). Pancreatic ductal adenocarcinoma: Biological hallmarks, current status, and future perspectives of combined modality treatment approaches. Radiation Oncology, 14(1), 141. https://doi.org/10.1186/s13014-019-1345-6

[2] Sarantis, P., Koustas, E., Papadimitropoulou, A., Papavassiliou, A. G., \& Karamouzis, M. V. (2020). Pancreatic ductal adenocarcinoma: Treatment hurdles, tumor microenvironment and immunotherapy. World Journal of Gastrointestinal Oncology, 12(2), 173-181. https://doi.org/10.4251/wjgo.v12.i2.173

[3] Norton, J., Foster, D., Chinta, M., Titan, A., \& Longaker, M. (2020). Pancreatic Cancer Associated Fibroblasts (CAF): Under-Explored Target for Pancreatic Cancer Treatment. Cancers, 12(5), 1347. https://doi.org/10.3390/cancers12051347

[4] Gao, Y., Gao, D., Shen, J., \& Wang, Q. (2020). A Review of Mesoporous Silica Nanoparticle Delivery Systems in Chemo-Based Combination Cancer Therapies. Frontiers in Chemistry, 8. https://doi.org/10.3389/fchem.2020.598722

[5] Prasanna, P. G. S., Stone, H. B., Wong, R. S., Capala, J., Bernhard, E. J., Vikram, B., \& Coleman, C. N. (2012). Normal tissue protection for improving radiotherapy: Where are the Gaps? Translational Cancer Research, 1(1), 35-48.

[6] Wang, S., Li, Y., Xing, C., Ding, C., Zhang, H., Chen, L., You, L., Dai, M., \& Zhao, Y. (2020). Tumor microenvironment in chemoresistance, metastasis and immunotherapy of pancreatic cancer. American Journal of Cancer Research, 10(7), $1937-1953$.

[7] Samanta, K., Setua, S., Kumari, S., Jaggi, M., Yallapu, M. M., \& Chauhan, S. C. (2019). Gemcitabine Combination Nano Therapies for Pancreatic Cancer. Pharmaceutics, 11(11), E574. https://doi.org/10.3390/pharmaceutics11110574

[8] Albrengues, J., Bertero, T., Grasset, E., Bonan, S., Maiel, M., Bourget, I., Philippe, C., Herraiz Serrano, C., Benamar, S., Croce, O., Sanz-Moreno, V., Meneguzzi, G., Feral, C. C., Cristofari, G., \& Gaggioli, C. (2015). Epigenetic switch drives the conversion of fibroblasts into proinvasive cancer-associated fibroblasts. Nature Communications, 6, 10204. https://doi.org/10.1038/ncomms10204

[9] Darby, I. A., Laverdet, B., Bonté, F., \& Desmoulière, A. (2014). Fibroblasts and myofibroblasts in wound healing. Clinical, Cosmetic and Investigational Dermatology, 7, 301-311. https://doi.org/10.2147/CCID.S50046

[10] Alkasalias, T., Moyano-Galceran, L., Arsenian-Henriksson, M., \& Lehti, K. (2018). Fibroblasts in the Tumor Microenvironment: Shield or Spear? International Journal of Molecular Sciences, 19(5). https://doi.org/10.3390/ijms19051532

[11] Ortiz-Otero, N., Clinch, A. B., Hope, J., Wang, W., Reinhart-King, C. A., \& King, M. R. (2020). Cancer associated fibroblasts confer shear resistance to circulating tumor cells during prostate cancer metastatic progression. Oncotarget, 11(12), 1037-1050. https://doi.org/10.18632/oncotarget.27510

[12] Nguyen, L., Schilling, D., Dobiasch, S., Raulefs, S., Santiago Franco, M., Buschmann, D., Pfaffl, M. W., Schmid, T. E., \& Combs, S. E. (2020). The Emerging Role of miRNAs for the Radiation Treatment of Pancreatic Cancer. Cancers, 12(12), E3703. https://doi.org/10.3390/cancers12123703

[13] Bannister, A., Dissanayake, D., Kowalewski, A., Cicon, L., Bromma, K., \& Chithrani, D. B. (2020). Modulation of the Microtubule Network for Optimization of Nanoparticle Dynamics for the Advancement of Cancer Nanomedicine. Bioengineering, 7(2). https://doi.org/10.3390/bioengineering7020056

[14] Bannister, A. H., Bromma, K., Sung, W., Monica, M., Cicon, L., Howard, P., Chow, R. L., Schuemann, J., \& Chithrani, D. B. (2020). Modulation of nanoparticle uptake, intracellular distribution, and retention with docetaxel to enhance radiotherapy. The British Journal of Radiology, 93(1106), 20190742. https://doi.org/10.1259/bjr.20190742 
[15] Bromma, K., Bannister, A., Kowalewski, A., Cicon, L., \& Chithrani, D. B. (2020). Elucidating the fate of nanoparticles among key cell components of the tumor microenvironment for promoting cancer nanotechnology. Cancer Nanotechnology, 11(1). https://doi.org/10.1186/s12645-020-00064-6

[16] Alhussan, A., Bromma, K., Bozdoğan, E. P. D., Metcalfe, A., Karasinska, J., Beckham, W., Alexander, A. S., Renouf, D. J., Schaeffer, D. F., \& Chithrani, D. B. (2021). Investigation of Nano-Bio Interactions within a Pancreatic Tumor Microenvironment for the Advancement of Nanomedicine in Cancer Treatment. Current Oncology, 28(3), 1962-1979. https://doi.org/10.3390/curroncol28030183

[17] Alhussan, A., Bromma, K., Perez, M. M., Beckham, W., Alexander, A. S., Howard, P. L., \& Chithrani, D. B. (2021). Docetaxel-Mediated Uptake and Retention of Gold Nanoparticles in Tumor Cells and in Cancer-Associated Fibroblasts. Cancers, 13(13), 3157. https://doi.org/10.3390/cancers13133157

[18] Bromma, K., Alhussan, A., Perez, M. M., Howard, P., Beckham, W., \& Chithrani, D. B. (2021). Three-Dimensional Tumor Spheroids as a Tool for Reliable Investigation of Combined Gold Nanoparticle and Docetaxel Treatment. Cancers, 13(6), 1465. https://doi.org/10.3390/cancers13061465

[19] Kasurinen, S., Happo, M. S., Rönkkö, T. J., Orasche, J., Jokiniemi, J., Kortelainen, M., Tissari, J., Zimmermann, R., Hirvonen, M.-R., \& Jalava, P. I. (2018). Differences between co-cultures and monocultures in testing the toxicity of particulate matter derived from $\log$ wood and pellet combustion. PLoS ONE, 13(2), e0192453. https://doi.org/10.1371/journal.pone.0192453

[20] Miki, Y., Ono, K., Hata, S., Suzuki, T., Kumamoto, H., \& Sasano, H. (2012). The advantages of co-culture over mono cell culture in simulating in vivo environment. The Journal of Steroid Biochemistry and Molecular Biology, 131(3-5), 68-75. https://doi.org/10.1016/j.jsbmb.2011.12.004

[21] Miller, A. J., \& Spence, J. R. (2017). In Vitro Models to Study Human Lung Development, Disease and Homeostasis. Physiology (Bethesda, Md.), 32(3), 246-260. https://doi.org/10.1152/physiol.00041.2016

[22] Goers, L., Freemont, P., \& Polizzi, K. M. (2014). Co-culture systems and technologies: Taking synthetic biology to the next level. Journal of the Royal Society Interface, 11(96), 20140065. https://doi.org/10.1098/rsif.2014.0065

[23] Wolfe, T., Chatterjee, D., Lee, J., Grant, J. D., Bhattarai, S., Tailor, R., Goodrich, G., Nicolucci, P., \& Krishnan, S. (2015). Targeted gold nanoparticles enhance sensitization of prostate tumors to megavoltage radiation therapy in vivo. Nanomedicine: Nanotechnology, Biology, and Medicine, 11(5), 1277-1283. https://doi.org/10.1016/j.nano.2014.12.016 